https://doi.org/10.15407/ujpe64.2.100

V.E. KUZMICHEV, V.V. KUZMICHEV

Bogolyubov Institute for Theoretical Physics,

Nat. Acad. of Sci. of Ukraine

(14-b, Metrolohichna Str., Kyiv 03143,Ukraine; e-mail: vkuzmichev@bitp.kiev.ua)

\title{
GENERALIZED UNCERTAINTY PRINCIPLE IN QUANTUM COSMOLOGY FOR THE MAXIMALLY SYMMETRIC SPACE
}

\begin{abstract}
The new uncertainty relation is derived in the context of the canonical quantum theory with gravity in the case of the maximally symmetric space. This relation establishes a connection between fluctuations of the quantities, which determine the intrinsic and extrinsic curvatures of the spacelike hypersurface in spacetime and introduces the uncertainty principle for quantum gravitational systems. The generalized time-energy uncertainty relation taking gravity into account gravity is proposed. It is shown that known Unruh's uncertainty relation follows, as a particular case, from the new uncertainty relation. As an example, the sizes of fluctuations of the scale factor and its conjugate momentum are calculated within an exactly solvable model. All known modifications of the uncertainty principle deduced previously from different approaches in the theory of gravity and the string theory are obtained as particular cases of the proposed general expression.

Keywords: quantum gravity, quantum geometrodynamics, cosmology, uncertainty principle.
\end{abstract}

\section{Introduction}

The Heisenberg uncertainty principle plays a fundamental role in quantum mechanics. It states that two observables that do not commute cannot be measured simultaneously with arbitrary accuracy $[1,2]$. A long time ago, it was recognized that the inclusion of the gravitational interaction into the fabric of quantum theory should lead to the modification of the Heisenberg uncertainty relation [3-6]. It is expected that, on scales less than Planck's scale, the classical concepts of space and time lose their meanings, and the radical revision of our notions of them is required. The minimum length of the order of Planck's length appears as a new ingredient of the theory with gravity and determines a natural restriction on measurable distances.

Possible modifications of the Heisenberg uncertainty relations, which consider the effects of gravity, have been debated since the middle of the 1980s. The modification of the position-momentum uncertainty relation for a test particle moving in a gravitational field was formulated, and the existence of a Planckscale minimal observable length was shown in string

(c) V.E. KUZMICHEV, V.V. KUZMICHEV, 2019 theory [7-9]. The same result was obtained, by using general model-independent properties of a quantum theory of gravitation $[10,11]$ with regard for the constraint on an upper limit of the acceleration of massive particles [12]. The consequences of the existence of a minimal length, the quantum-mechanical structure, which underlies it [13] and its effect on the physical properties of various objects (the hydrogen atom spectrum [14], the Lamb shift, Landau levels, and others [15]) were investigated (an extensive bibliography can be found, e.g. in Refs. [16-19]). The existence of a minimum observable momentum can lead to a more general understanding of the influence of gravity on the dynamics of the system [20].

It seems indubitable that, for the clarification of the influence of spacetime curvature effects on the magnitude of dispersions of two observables corresponding to canonically conjugate variables (such as position and momentum, time and energy), one should have a quantum theory at its disposal, which treats gravity on the same grounds as quantized matter fields. A consistent quantum theory of gravity, in principle, can be constructed on the basis of the ArnowittDeser-Misner (ADM) Hamiltonian formalism [21] of general relativity with the application of the canon-

ISSN 2071-0194. Ukr. J. Phys. 2019. Vol. 64, No. 2 
ical quantization method. The canonical approach (which is successful in constructing the nonrelativistic quantum mechanics and quantum field theories in the flat spacetime) to the quantization encounters well-known difficulties, when applied to gravity, such as the understanding of the time evolution, the divergence of the norm of the state vectors, the measurement problem, and others. The structure of constraints in general relativity is such that the variables corresponding to the true dynamical degrees of freedom cannot be singled out. This is stipulated by the absence of a predetermined way to identify spacetime events in generally covariant theory [22]. The quantum theory of gravity based on the Wheeler-DeWitt equation makes no reference to a time parameter $[23,24]$. Attempts to identify one of the matter field variables of the equations of quantum geometrodynamics with time were not successful. However, time can be introduced in the theory in a special manner by bringing in an additional matter source (similar to DeWitt's relativistic elastic media with clocks) in the form of a perfect (reference) fluid, which defines a dynamical reference system (material reference frame) $[25,26]$.

A model with a finite number of degrees of freedom may provide a reasonable framework for addressing the problems of quantum gravity. The homogeneous minisuperspace models have been proven to be successful - consistent with observations and having predictive power - in classical cosmology. This appears explicable, in view of the fact that the Universe can, to first approximation, be considered as being homogeneous, and gives rise to the hope for that homogeneous models could be useful in quantum cosmology as well. For such models, the quantum theory of gravity with a well-defined time variable was proposed and studied in Refs. [27-31].

In the present paper, we study the fluctuations of the observables that characterize the quantum gravitational system as such, like the intrinsic and extrinsic curvatures of a spacelike hypersurface in spacetime, and the influence of gravity on quantum fluctuations of the position and the momentum of motion of a test particle in coordinate space. In order to formulate the uncertainty relation, we require well-defined state vectors, which will allow us to calculate the expectation values of observables and their statistical fluctuations. In Sect. 2, the version of quantum theory of a gravitational system in the maximally symmetric space is given. The distinguished feature of this theory is that one quantizes the observable represented by some Hermitian operator, which plays the role of an effective Hamiltonian and is measured in units of the conversion constant $(\hbar c)$. Therefore, no difficulties with the definition of energy in general relativity and in corresponding quantum field theory constructed on its basis arise in this approach. Sect. 3 is devoted to the derivation of the uncertainty principle for quantum systems with the gravitational interaction. It is shown that the Heisenberg uncertainty relations can be reformulated in terms of geometrical values such as intrinsic and extrinsic curvatures. In Sect. 4, the new generalized uncertainty relation of energy and time is formulated, and its connection with the geometrical variables of the theory is shown. The goal of Sect. 5 is to reduce the obtained uncertainty relation for quantum gravitational systems to Unruh's relation between the metric and the curvature. In Sect. 6, the fluctuations of cosmological parameters are calculated within an exactly solvable model. In Sect. 7, the uncertainty relation, which takes the gravitational interaction into account, for fluctuations of the position and momentum of a test particle is obtained. This relation yields the generalized uncertainty principle proposed previously by a number of authors, by using different quantum theories with gravity. The consequences of the existence of the minimum measurable length and momentum of a test particle are also discussed here. Finally, conclusions are drawn in Sect. 8.

\section{Quantization Scheme}

Consider the homogeneous isotropic quantum gravitational system (QGS). In the case of maximally symmetric geometry with the Robertson-Walker metric, the geometrical properties of the system are determined by a single variable, namely the cosmic scale factor $a$. The matter sector of the QGS is taken in the form of a uniform scalar field $\phi$ with a self-adjoint Hamiltonian $H_{\phi}$. This Hamiltonian is defined in a curved spacetime and depends on the scale factor $a$ as a parameter, $H_{\phi}=H_{\phi}(a)$. The scalar field can be interpreted as a surrogate of all possible real physical fields of matter averaged with respect to the spin, space, and other degrees of freedom. In addition, it is accepted that the QGS is filled with a perfect fluid in the form of a relativistic matter with the energy 
density $\rho_{\gamma}=E / a^{4}$, where $E$ is constant. A perfect fluid coupled to a fleet of clocks can be employed as a material reference frame enabling one to recognize the instants of time. Following Dirac's approach to quantum gravity [32], we do not solve constraints prior to the quantization, but convert the secondclass constraints into the first-class ones, which become constraints on the state vector (wave function) $\langle a, \phi \mid \Psi(T)\rangle$ in the representation of the gravitational and matter fields, $a$ and $\phi$, the parameter $T$ is a conformal time. The basic equations of such a QGS appear as the set of two partial differential equations $[27-31]^{1}$,

$$
\begin{aligned}
& \left(-i \partial_{T}-\frac{1}{2} E\right)|\Psi(T)\rangle=0 \\
& \left(-\partial_{a}^{2}+\kappa a^{2}-2 a H_{\phi}-a^{4} \frac{\Lambda}{3}-E\right)|\Psi(T)\rangle=0
\end{aligned}
$$

where the curvature constant $\kappa=+1,0,-1$ for spatially closed, flat, and open QGSs, respectively, and $\Lambda$ is the cosmological constant. The conformal time $T$ is expressed in radians, the cosmic scale factor $a$ is measured in units of Planck's length $l_{\mathrm{P}}=\sqrt{2 G \hbar /\left(3 \pi c^{3}\right)}$, $G$ is Newton's gravitational constant, the Hamiltonian $H_{\phi}$ is taken in the units of Planck's energy $m_{\mathrm{P}} c^{2}=\hbar c / l_{\mathrm{P}}$, the energy density $\rho_{\gamma}$ is in the units of Planck's density $\rho_{\mathrm{P}}=3 c^{4} /\left(8 \pi G l_{\mathrm{P}}^{2}\right)$, so that $E$, which has the dimension of [Energy $\times$ Length] $=[\hbar c]$ in ordinary physical units, becomes dimensionless. In such units, the commutation relation between $a$ and its conjugate momentum $\pi=-i \hbar \partial_{a}$ takes the form

$\left[a,-i \partial_{a}\right]=i$.

Equations (1) can be combined into a single Schrödinger-type time equation

$-i \partial_{T}|\Psi(T)\rangle=\mathrm{H}|\Psi(T)\rangle$,

where the operator

$\mathrm{H}=\frac{1}{2}\left(-\partial_{a}^{2}+\kappa a^{2}-2 a H_{\phi}-a^{4} \frac{\Lambda}{3}\right)$

can be considered as the effective Hamiltonian of the QGS, which does not depend on the time $T$ explicitly,

\footnotetext{
${ }^{1}$ Here, for simplicity, the rescaling of the variable $T$ is done, and the corresponding coefficient is included into the definition of proper time.
}

and the minus sign is a consequence of the gravitational field equations in general relativity. The condition of self-adjointness of $H_{\phi}$ and the reality of $a$ yield the self-adjointness of $\mathrm{H}$.

Let us define the unitary evolution operator

$U\left(T, T_{0}\right)=e^{i \mathrm{H}\left(T-T_{0}\right)}$,

where $T_{0}$ is an arbitrary constant taken as a time reference point. Then the solution of Eq. (3) can be written as follows:

$|\Psi(T)\rangle=U\left(T, T_{0}\right)\left|\Psi_{\mathrm{H}}\left(T_{0}\right)\right\rangle$

where $\left|\Psi_{\mathrm{H}}\left(T_{0}\right)\right\rangle$ is a state vector in the Heisenberg representation.

The second equation from set (1), which defines a change of the state vector $|\Psi(T)\rangle$ as a function of $a$ and $\phi$, can be integrated with respect to $\phi$. With this purpose, we introduce the complete set of orthonormalized functions $\left\langle\chi \mid u_{k}\right\rangle$ in a representation of the rescaled variable $\chi=\chi(a, \phi)$, in which the Hamiltonian $H_{\phi}$ is diagonalized,

$\left\langle u_{k}\left|H_{\phi}\right| u_{k^{\prime}}\right\rangle=M_{k}(a) \delta_{k k^{\prime}}$,

where $M_{k}(a)$ is the proper mass-energy of the new effective matter in the discrete and/or continuous $k$ th state obtained after the averaging of $H_{\phi}$ with respect to the field $\chi$ in a comoving volume $\frac{1}{2} a^{3}$. For example, in the case where $H_{\phi}$ describes the homogeneous scalar field, the new effective matter is a barotropic fluid with the energy density $\rho_{m}=2 M_{k}(a) / a^{3}$ and the pressure $p_{m}=-\frac{1}{3}\left(d \ln M_{k}(a) / d \ln a\right) \rho_{m}$ [30].

Further, we bring in another complete set of orthonormalized functions $\left\langle a \mid f_{n k}\right\rangle$, which satisfies the equation

$\left(-\partial_{a}^{2}+\kappa a^{2}-2 a M_{k}(a)-a^{4} \frac{\Lambda}{3}\right)\left|f_{n k}\right\rangle=E_{n}\left|f_{n k}\right\rangle$,

where $n$ enumerates discrete and/or continuous states of the QGS with matter in the fixed $k$ th state. Then the vector $\left|\Psi_{\mathrm{H}}\left(T_{0}\right)\right\rangle$ can be written as the superposition

$\left|\Psi_{\mathrm{H}}\left(T_{0}\right)\right\rangle=\sum_{n, k} C_{n k}\left(T_{0}\right)\left|u_{k}\right\rangle\left|f_{n k}\right\rangle$,

where the coefficient $C_{n k}\left(T_{0}\right)$ gives the probability $\left|C_{n k}\left(T_{0}\right)\right|^{2}$ to find the QGS in the $n$th state of relativistic matter and the $k$ th state of averaged effective

ISSN 2071-0194. Ukr. J. Phys. 2019. Vol. 64, No. 2 
matter at the instant of time $T_{0}$. Since, by definition, the vectors $\left|u_{k}\right\rangle$ and $\left|f_{n k}\right\rangle$ exhaust all the possible states of the matter components of the QGS, the normalization condition for $|\Psi(T)\rangle$ takes the form

$$
\begin{aligned}
& \langle\Psi(T) \mid \Psi(T)\rangle=\left\langle\Psi_{\mathrm{H}}\left(T_{0}\right) \mid \Psi_{\mathrm{H}}\left(T_{0}\right)\right\rangle= \\
& =\sum_{n, k}\left|C_{n k}\left(T_{0}\right)\right|^{2}=1 .
\end{aligned}
$$

The transition amplitude $T_{0} \rightarrow T$ determines the mean value of the evolution operator with respect to state $(9)$,

$$
\begin{aligned}
& \left\langle\Psi\left(T_{0}\right) \mid \Psi(T)\right\rangle=\left\langle\Psi_{\mathrm{H}}\left(T_{0}\right)\left|U\left(T, T_{0}\right)\right| \Psi_{\mathrm{H}}\left(T_{0}\right)\right\rangle= \\
& =\sum_{n, k} e^{\frac{i}{2} E_{n}\left(T-T_{0}\right)}\left|C_{n k}\left(T_{0}\right)\right|^{2} .
\end{aligned}
$$

It demonstrates that the evolution of the system in time $T$ has a periodic character in each $n$th state. In view of the self-adjointness of the effective Hamiltonian (4), Eq. (3) and its complex conjugate yield the equation

$\frac{d\langle\mathrm{~A}\rangle}{d T}=\frac{1}{i}\langle[\mathrm{H}, \mathrm{A}]\rangle+\left\langle\frac{\partial \mathrm{A}}{\partial T}\right\rangle$,

which gives the time-dependence of the mean value of an observable A,

$$
\begin{aligned}
& \langle\mathrm{A}\rangle \equiv\langle\Psi(T)|\mathrm{A}| \Psi(T)\rangle= \\
& =\left\langle\Psi_{\mathrm{H}}\left(T_{0}\right)\left|\mathrm{A}_{\mathrm{H}}\right| \Psi_{\mathrm{H}}\left(T_{0}\right)\right\rangle \equiv\left\langle\mathrm{A}_{\mathrm{H}}\right\rangle_{\mathrm{H}},
\end{aligned}
$$

where $\mathrm{A}_{\mathrm{H}}=U^{\dagger} \mathrm{A} U$ is the observable of the Heisenberg representation. From the unitary property of the operator $U(5)$, we have

$\langle[\mathrm{H}, \mathrm{A}]\rangle=\left\langle\left[\mathrm{H}_{\mathrm{H}}, \mathrm{A}_{\mathrm{H}}\right]\right\rangle_{\mathrm{H}}$,

where $\mathrm{H}_{\mathrm{H}}=U^{\dagger} \mathrm{H} U$ is the Hamiltonian of the Heisenberg representation.

Let us note that, according to Eq. (9), Eqs. (7) and (8) determine the stationary quantum states of the QGS at some fixed instant of time $T_{0}$, the choice of which is arbitrary, $\left\langle\chi \mid u_{k}\right\rangle \equiv u_{k}\left(\chi, T_{0}\right)$, and $\left\langle a \mid f_{n k}\right\rangle \equiv$ $\equiv f_{n k}\left(a, T_{0}\right)$.

\section{Uncertainty Principle}

The uncertainty relation for two observables in a Hilbert space, A and B, which do not depend on the time $T$, can be explicitly written as (cf. Ref. [2])

$\Delta \mathrm{A} \Delta \mathrm{B} \geq \frac{1}{2}|\langle[\mathrm{~A}, \mathrm{~B}]\rangle|$, where $\Delta \mathrm{A}=\sqrt{\left\langle\mathrm{A}^{2}\right\rangle-\langle\mathrm{A}\rangle^{2}}, \Delta \mathrm{B}=\sqrt{\left\langle\mathrm{B}^{2}\right\rangle-\langle\mathrm{B}\rangle^{2}}$ are the root-mean-square deviations of $\mathrm{A}$ and $\mathrm{B}$, respectively.

Let $\mathrm{A}=a$ and $\mathrm{B}=\pi=-i \partial_{a}$. Then, taking Eq. (2) into account, we find the uncertainty relation between the scale factor and its conjugate momentum:

$\Delta a \Delta \pi \geq \frac{\hbar}{2}$

(in ordinary physical units). This relation coincides in form with the uncertainty relation between the position and the momentum in ordinary quantum mechanics, but it has a different physical meaning. It is expressed in geometrical quantities and thus describes the effects of spacetime curvature.

Inequality (16) reduces to an equality, if $\Delta a=l_{\mathrm{P}}$ and $\Delta \pi=\frac{1}{2} m_{\mathrm{P}} c$. It can be considered as an indication of the existence of a minimum length equal to Planck's length. Really, we have $\Delta a \rightarrow 0$, so that quantum fluctuations $\Delta \pi \rightarrow \infty$ near the cosmological singularity $a=0$. This means that, at distances shorter than Planck's length, there should exist a limitation on the measurement of spacetime quantities. The existence of a minimal measurable length of the order of Planck's length (quantitative limit to spacetime resolution) was predicted in the 1960s [33] (for details, see Refs. [11,34]). Below, in Sects. 6 and 7 , we give a calculation of the minimum length and minimum momentum on specific examples.

The uncertainty relation (16) establishes, in fact, a connection between fluctuations of the quantities, which determine the intrinsic and extrinsic curvatures of a spacelike hypersurface in spacetime. By associating quantum operators to the scalar curvature ${ }^{(3)} R$ and the extrinsic curvature tensor $K_{i j}=$ $=-\frac{1}{2} \partial^{(3)} g_{i j} / \partial \tau$, where ${ }^{(3)} g_{i j}$ is the 3 -metric and $\tau$ is the proper time, Eq. (16) can be written explicitly in terms of curvature fluctuations,

$\Delta^{(3)} R \Delta K \gtrsim 4 \pi \hbar \frac{\left|{ }^{(3)} R\right|}{{ }^{(3)} V}$,

where $K=K_{i}^{i}$, and ${ }^{(3)} V \sim \frac{4}{3} \pi a^{3}$ is the 3 -volume of the measurement (observed part of the QGS).

\section{Generalized Time-Energy Uncertainty Relation}

Let us find the generalized time-energy uncertainty relation, which takes gravity into account. In the 
quantum gravitational system, let A be an observable, which does not depend on time explicitly and does not commute with the effective Hamiltonian (4). Setting $\mathrm{B}=\mathrm{H}$ in Eq. (15), bearing in mind Eq. (12) and that the proper time $\tau$ is connected with the conformal time $T$ by the differential equation $c d \tau=a d T$, we obtain the uncertainty relation in ordinary physical units:

$\tau_{\mathrm{A}} \frac{\Delta \mathrm{E}}{|a|} \geq \frac{\hbar}{2}$

where $\Delta \mathrm{E}$ is the root-mean-square deviation of $\mathrm{H}$, and

$\tau_{\mathrm{A}}=\left|\Delta \mathrm{A}\left(\frac{d\langle\mathrm{~A}\rangle}{d \tau}\right)^{-1}\right|$

is a time characteristic of the evolution of the statistical distribution of A (i.e. the time necessary for this statistical distribution to be considerably modified). The quantity $\Delta \mathrm{E} /|a|$ is the statistical fluctuation of the result of the energy measurement, where the denominator involves the redshift correction due to the expansion of the QGS. In the limit $|a| \rightarrow 0$, this fluctuation becomes infinitely large, while a time characteristic $\tau_{\mathrm{A}}$ can acquire any value in accordance with the uncertainty relation (18). Thus, near the initial cosmological singularity, the notions of time and energy lose their meaning (they cannot be measured).

Because of the presence of the multiplier $1 /|a|$, the uncertainty relation (18) does not reduce to the timeenergy uncertainty relation of ordinary quantum mechanics. In our approach, the effective Hamiltonian has the dimension of the constant $E$, which is quantized according to Eq. (8), the latter determines the quantum state of relativistic matter.

For $\mathrm{A}=a$, relation (18) reduces to the uncertainty relation (16), where $\Delta \pi=\frac{\Delta \mathrm{E}}{c}\left(\frac{d\langle a\rangle}{d T}\right)^{-1}$ is the statistical fluctuation of the momentum.

\section{Unruh's Uncertainty Relation}

The uncertainty relation (16) can be reduced to Unruh's uncertainty relation between the metric and the curvature. One may assume formally that the Einstein's equations are valid in the quantum regime as well [35]. Indeed, as is shown in Ref. [29], Eq. (12) for $\mathrm{A}=a$ and $\mathrm{A}=\pi=-i \partial_{a}$ can be reduced to the Einstein-Friedmann equations, which contain the quantum correction terms to the total energy density and pressure. Then the rate of change of the momentum in time is given by the equation $\dot{\pi}=-\frac{1}{2} a^{2} T_{\alpha}^{\alpha}+\kappa$, where $T_{\alpha}^{\alpha}$ is the trace of the stress tensor. We shall restrict ourselves to the study of the fluctuations of the quantities in spatial directions. In a comoving reference frame, one can express the $T_{x}^{x}$ component of the stress tensor as $T_{x}^{x}=-\mathrm{p}$, where $\mathrm{p}$ is the pressure defined as the force acting on the surface element having an area of $A$ in the direction of $x$. In that case, the fluctuation of the momentum can be estimated as $\Delta \pi \sim \Delta T_{x}^{x} A \delta \tau$, where $\delta \tau$ is a time interval, $A \sim a^{2}$ and $\Delta T_{x}^{x} \sim T_{x}^{x}$. The metric component $g_{x x}$ can be represented in the form $g_{x x}=a^{2} \gamma_{x x}$, where $\gamma_{x x}$ is the comoving spatial metric component, whose fluctuation can be neglected, $\Delta \gamma_{x x}=0$. Then the fluctuations $\Delta g_{x x}$ and $\Delta a$ are connected between themselves: $\Delta g_{x x} / g_{x x}=2 \Delta a / a$. As a result, in the rest frame, relation (16) takes the form

$\Delta g_{x x} \Delta T_{x}^{x} \gtrsim \hbar \frac{g_{x x}}{\delta \tau^{(3)} V}$,

where ${ }^{(3)} V \sim a^{3}$ is the 3 -volume. Introducing the Einstein tensor, $G_{x}^{x}=8 \pi T_{x}^{x}$ (in units $G=c=1$ ) and defining the 4 -volume ${ }^{(4)} V \sim \delta \tau^{(3)} V$, we rewrite the preceding relation in Unruh's form

$\Delta g_{x x} \Delta G^{x x} \gtrsim \hbar \frac{8 \pi}{(4) V}$.

From Eq. (21), the Heisenberg position-momentum uncertainty relation, which considers the effects of gravity on quantum fields, can be restored (cf. Ref. [35]).

Equation (20) can be represented also as

$\delta \varepsilon \delta \tau \gtrsim \hbar$

which connects a deviation of the energy $\delta \varepsilon=\Delta g_{x x} \times$ $\times \Delta T^{x x(3)} V$ with the time interval $\delta \tau$ (cf. with Eq. (18)).

\section{Exactly Solvable Model}

The uncertainty relation (16) and its consequences (17)-(22) determine restrictions on the simultaneous measurement of the corresponding cosmological parameters of the QGS described by the state vector $|\Psi(T)\rangle$. According to Eqs. (6) and (9), such a QGS is a superposition of subsystems (or universes in a multiverse model) characterized by the quantum numbers $k$ and $n$, i.e., they are filled with the effective

ISSN 2071-0194. Ukr. J. Phys. 2019. Vol. 64, No. 2 
matter in the $k$ th state with definite mass-energy $M_{k}(a)$ and relativistic matter in the $n$th state with the energy $E_{n} /(2 a)$. Each subsystem is described by its wave function $\left\langle a \mid f_{n k}\right\rangle$, which satisfies the stationary equation (8). The time equation for $\left|f_{n k}(T)\right\rangle=$ $=e^{\frac{i}{2} E_{n}\left(T-T_{0}\right)}\left|f_{n k}\right\rangle$ follows from Eqs. (3), (6), and (9):

$-i \partial_{T}\left|f_{n k}(T)\right\rangle=\mathrm{H}_{k}\left|f_{n k}(T)\right\rangle$,

with the "Hamiltonian"

$\mathrm{H}_{k}=\frac{1}{2}\left(-\partial_{a}^{2}+\kappa a^{2}-2 a M_{k}(a)-a^{4} \frac{\Lambda}{3}\right)$,

which is operator (4) averaged over the states $\left|u_{k}\right\rangle$, $\mathrm{H}_{k}=\left\langle u_{k}|\mathrm{H}| u_{k}\right\rangle$.

The uncertainty relation for the observables $a$ and $\pi=-i \partial_{a}$ in such a subsystem takes the form

$\delta a \delta \pi \geq \frac{1}{2}$

(in dimensionless units), where $\delta a=\sqrt{\left\langle a^{2}\right\rangle_{n k}-\langle a\rangle_{n k}^{2}}$ and $\delta \pi=\sqrt{\left\langle\pi^{2}\right\rangle_{n k}-\langle\pi\rangle_{n k}^{2}}$ are the root-mean-square deviations, and the averaging is performed over the $f_{n k}$-states, e.g. $\left\langle a^{2}\right\rangle_{n k} \equiv\left\langle f_{n k}\left|a^{2}\right| f_{n k}\right\rangle$ and so on.

Let us calculate the fluctuations $\delta a$ and $\delta \pi$ in an explicit form for a specific spatially closed subsystem with zero cosmological constant $(\Lambda=0)$ filled with separate (non-interacting) macroscopic bodies (dust) and radiation. The mass of the dust is $M_{k}(a)=$ $=\mu\left(k+\frac{1}{2}\right)=$ const, where $\mu$ is the mass of a single macroscopic body, and $k$ is the number of such bodies. In this case, the solution of Eq. (8) is

$$
\begin{aligned}
& \left|f_{n k}\right\rangle \equiv f_{n}\left(\xi_{k}\right)=N_{n k} e^{-\frac{1}{2} \xi_{k}^{2}} H_{n}\left(\xi_{k}\right), \\
& E_{n}=2 n+1-M_{k}^{2},
\end{aligned}
$$

where $\xi_{k}=a-M_{k}, H_{n}$ is the Hermite polynomial, $N_{n k}$ is the normalizing constant, and the wave function is normalized on the interval $\left[-M_{k}, \infty\right)$.

Then, with an accuracy of order $e^{-M_{k}^{2}}[27]$, we have

$$
\left\langle a^{2}\right\rangle_{n k}=n+\frac{1}{2}+M_{k}^{2}, \quad\langle a\rangle_{n k}=M_{k}
$$

so that (in ordinary physical units)

$\delta a=l_{\mathrm{P}} \sqrt{n+\frac{1}{2}}$.
For the momentum, we obtain

$$
\left\langle\pi^{2}\right\rangle_{n k}=n+\frac{1}{2}, \quad\langle\pi\rangle_{n k}=0
$$

and

$\delta \pi=m_{\mathrm{P}} c \sqrt{n+\frac{1}{2}}$.

As a consequence, we get the uncertainty product of the same form as for a harmonic oscillator,

$\delta a \delta \pi=\left(n+\frac{1}{2}\right) \hbar$

From Eqs. (28) and (30), one can see that the fluctuations $\delta a$ and $\delta \pi$ take minimum values in the ground (vacuum) state with $n=0$,

$\delta a_{\min }=\frac{l_{\mathrm{P}}}{\sqrt{2}}, \quad \delta \pi_{\min }=\frac{m_{\mathrm{P}} c}{\sqrt{2}}$.

The size of fluctuations increases as the square root $\sqrt{n}$. It is interesting to estimate the size of fluctuations in the subsystem having the mass-energy of the observable part of our universe $l \sim 10^{28} \mathrm{~cm}$. The cosmological parameters $E_{n} \sim 10^{118} \ll M_{k}^{2} \sim 10^{122}$ (i.e. $\rho_{\gamma} \sim 10^{-10} \mathrm{GeV} \mathrm{cm}^{-3}$ and $\rho_{m} \sim 10^{-5} \mathrm{GeV} \mathrm{cm}^{-3}$ ) correspond to $n \sim 10^{122}$ and fluctuations $\delta a \sim l \sim$ $\sim 10^{28} \mathrm{~cm}$. In such a description, the observable part of the universe appears as a gigantic fluctuation.

\section{Test Particle in Gravitational Field}

In the preceding sections, we have analyzed the properties of a quantum gravitational system in connection with uncertainty relations. Here, we will consider an influence of gravity on quantum fluctuations of the position and momentum of a test particle. By introducing the root-mean-square deviations of the position $\Delta x$ and momentum $\Delta p$ of motion of a test particle in the coordinate space, one can rewrite the uncertainty relation (16) in the form, which takes the effects of gravity on motion into account:

$\Delta x \frac{\Delta p}{\hbar} \geq \frac{1}{2} \frac{\Delta x}{\Delta a} \frac{\Delta p}{\Delta \pi}$.

We suppose that the deviations $\Delta a$ and $\Delta \pi$ can be represented as follows:

$\Delta a=\langle\rho(Q)\rangle \Delta x, \quad \Delta \pi=\langle\zeta(P)\rangle \Delta p$, 
where $\rho(Q)$ and $\zeta(P)$ are some functions of deviations of the position $Q=x-\langle x\rangle$ and the momentum $P=$ $=p-\langle p\rangle$, and the averaging ensures the independence of $\Delta a$ and $\Delta \pi$ from direction in the coordinate space.

In view of the random character of the fluctuations of the result of measurement of the position and momentum of a test particle around their average values, let us define the functions $\rho(Q)$ and $\zeta(P)$ in the form of the normal frequency functions

$\rho(Q)=e^{-\frac{Q^{2}}{2 L_{x}^{2}}}, \quad \zeta(P)=e^{-\frac{L_{p}^{2}}{2}\left(\frac{P}{\hbar}\right)^{2}}$

normalized to satisfy $\rho(0)=1, \zeta(0)=1$. Here, $L_{x}^{2}$ is the dispersion in the coordinate space, and $\left(\frac{\hbar}{L_{p}}\right)^{2}$ is the dispersion in the momentum space.

The normal distributions (35) correspond to the wave packet representing a test particle localized in the coordinate space about $Q=0$ and localized in the momentum space about $P=0$. The quantities $L_{x}$ and $L_{p}$ have the dimensions of length. They are independent free parameters, since the normal frequency function $\zeta(P)$ is not a Fourier transform of $\rho(Q)$. The physical contents of the parameters $L_{x}$ and $L_{p}$ are different. As is obvious already from Eqs. (33)(35), if the fluctuations $\Delta x$ and $\Delta p$ are non-zero, the Heisenberg uncertainty relation can be restored only in the formal limits $L_{x} \rightarrow \infty$ and $L_{p} \rightarrow 0$ reached simultaneously. If $L_{x}=\infty$, but $L_{p} \neq 0$, then gravity contributes to fluctuations of the momentum. In the other case, if $L_{x}<\infty$, but $L_{p}=0$, then gravity affects fluctuations of the position.

The reason why the parameters $L_{x}$ and $L_{p}$ have different physical contents is that the position $x$ and momentum $p$ fluctuate independently under the action of gravity. The spacetime and momentum space are both dynamical and fluctuating, and the momentum space is independent of the spacetime and it cannot be just a Fourier transform of the coordinate space (cf. Refs. [36, 37]).

The uncertainty relation (33) with deviations $\Delta a$ and $\Delta \pi(34)$ and the normal frequency functions (35) transforms into the generalized Heisenberg-type uncertainty relation with corrections to gravity, in the case

$\left(\frac{Q}{L_{x}}\right)^{2}<1$ and $\left(\frac{L_{p} P}{\hbar}\right)^{2}<1$.

Substituting Eq. (34) and Eq. (35) into Eq. (33), expanding the exponentials in Eq. (35) in power series, and truncating after the first corrections to unity, we obtain the approximate inequality

$\Delta x \geq \frac{1}{2}\left[\left(1+\frac{\Lambda}{6}(\Delta x)^{2}\right) \frac{\hbar}{\Delta p}+\right.$

$\left.+\frac{G}{2 c^{3}}\left(1+\frac{\Lambda}{6}(\Delta x)^{2}\right) \Delta p\right]$

where it is taken that $L_{p}=\sqrt{\frac{G \hbar}{c^{3}}}$ is Planck's length and $L_{x}=\sqrt{\frac{3}{\Lambda}}$ is the de Sitter horizon [20], and $\Lambda$ is a cosmological constant. The right-hand side of inequality (37) contains only the fundamental constants. From this inequality, under the assumption $\Lambda=0$, we get the result obtained in Refs. [10, 12]:

$\Delta x \geq \frac{\hbar}{2 \Delta p}+\frac{G}{2 c^{3}} \Delta p$.

This relation can be written in the form arising from string theory $[8,9]$ as

$\Delta x \geq \frac{\hbar}{2 \Delta p}+\frac{\alpha^{\prime}}{2} \frac{\Delta p}{\hbar}$,

where $\alpha^{\prime}=L_{p}^{2}$ is a fundamental constant controlling the tension of a string. The minimum length in such a theory is equal to $(\Delta x)_{\min }=\sqrt{\alpha^{\prime}}$ and coincides with Planck's length $\sim 10^{-33} \mathrm{~cm}$. From Eq. (37), it follows that there should exist not only the minimum length $(\Delta x)_{\min }=L_{p}$, but also the minimum momen$\operatorname{tum}(\Delta p / \hbar)_{\min }=1 / L_{x}$. If $L_{x}$ is the de Sitter horizon, then $(\Delta p / \hbar)_{\min } \sim \sqrt{\Lambda} \lesssim 10^{-28} \mathrm{~cm}^{-1}$ for the presentday values of the cosmological parameters.

By taking the minimum length together with the minimum momentum, one can write the following relation: $(\Delta x)_{\min }(\Delta p / \hbar)_{\min }=L_{p} / L_{x} \lesssim 10^{-61}$. Using this relation, it is possible to restore the physical parameters $A_{\text {today }}$ of the observed part of our Universe from the Planck values $A_{\text {Planck }}, A_{\text {today }}=$ $=A_{\text {Planck }}(\Delta x)_{\min }^{-1}(\Delta p / \hbar)_{\text {min }}^{-1}$. Substituting the Planck length, Planck mass, and Planck time into this expression, we obtain the estimations for the size of the observed part of the Universe $R_{0} \gtrsim 10^{28} \mathrm{~cm}$, for the mass $M_{0} \gtrsim 10^{80} \mathrm{GeV}$, and for the age of the Universe $t_{0} \gtrsim 10^{17} \mathrm{~s}$.

\section{Conclusion}

In this paper, we have studied the Heisenberg uncertainty principle in the context of the quantum geometrodynamics in the case of the maximally symmetric space. The obtained uncertainty principle in

ISSN 2071-0194. Ukr. J. Phys. 2019. Vol. 64, No. 2 
the form of Eq. (16) coincides in form with the uncertainty relation between a position and a momentum in ordinary quantum mechanics, but it is expressed in geometrical quantities and thus describes the effects of spacetime curvature. As is shown in Eq. (17), the uncertainty relation can be reformulated through the quantities, which determine the intrinsic and extrinsic curvatures of a spacelike hypersurface in the spacetime. It is found that the product of fluctuations of the intrinsic and extrinsic curvatures must be greater than or of order the intrinsic curvature per unit volume of the measurement.

Since the version of quantum theory of the gravitational system discussed in the paper contains a welldefined time variable, we are able to derive the generalized time-energy uncertainty relation (18), which contains the statistical fluctuation of the result of the measurement of the energy of the relativistic matter and includes the correction due to the expansion of the system. The obtained relation confirms a conjecture, which was previously considered in the literature, that the notions of time and energy lose their meaning near the initial cosmological singularity.

Under the assumption that the Einstein equations (with quantum correction terms) are valid in the quantum regime, we have demonstrated that the fundamental relation (16) can be reduced to the uncertainty relation in Unruh's form (21). Such a connection between Eqs. (16) and (21) may be interpreted as clarifying the physical meaning of Eq. (21).

Then we consider an exactly solvable model in the context of the problem of existence of the minimum physical length and momentum. Equations (3)-(9) describe the QGS as a linear superposition of simpler subsystems, each of which is characterized by matter-energy in specific states with quantum numbers $k$ and $n$. Each separate subsystem is determined by the wave function $\left\langle a \mid f_{n k}\right\rangle$ satisfying Eq. (8). If the subsystem is spatially closed and filled with dust and relativistic matter, then, in the case of zero cosmological constant, Eq. (8) has the analytic solution (26) in the form of an oscillator shifted in the $a$ axis by the amount of dust mass. The fluctuations of the scale factor $\delta a$ and its conjugate momentum $\delta \pi$ are quantized according to Eqs. (28) and (30), while their product (31) satisfies the uncertainty relation (25), where the equality is reached only for the vacuum state of the subsystem. In the vacuum state, the fluctuation $\delta a$ has the minimum value of the order of
Planck's length $l_{\mathrm{P}}$, and the fluctuation $\delta \pi$ acquires the minimum value of the order of Planck's momentum $m_{\mathrm{P}} c$ (see Eq. (32)).

Finally, we have studied the influence of gravity on quantum fluctuations of the position and momentum of a test particle. With regard for the random character of the fluctuations of the result of measurement of the position and momentum of a test particle around their average values, the uncertainty relation (33) transforms into the generalized Heisenbergtype uncertainty relation with corrections to gravity (37). From this inequality, the generalized uncertainty principle proposed previously by a number of authors can be reproduced.

The present work was partially supported by the National Academy of Sciences of Ukraine (projects Nos. 0117U00237 and 0116U003191).

1. L. de Broglie. Les Incertitudes d'Heisenberg et l'Interprétation Probabiliste de la Mécanique Ondulatoire (Gauthier-Villars, 1982) [ISBN: 978-2040154110].

2. A. Messiah. Quantum Mechanics (North Holland, 1981) [ISBN: 978-0720400441].

3. M. Bronstein. Quantentheorie schwacher Gravitationsfelder. Phys. Z. Sowjetunion 9, 140 (1936).

4. M. Bronstein. Quantization of gravitational waves. ZhETF 6, 195 (1936).

5. E.P. Wigner. Relativistic invariance and quantum phenomena. Rev. Mod. Phys. 29, 255 (1957).

6. J.L. Anderson. Quantization of general relativity. In: Gravitation and Relativity, ed. by H-Y. Chiu, W.F. Hoffmann, (Benjamin, 1964).

7. D. Amati, M. Ciafaloni, G. Veneziano. Can spacetime be probed below the string size? Phys. Lett. B 216, 41 (1989).

8. K. Konishi, G. Paffuti, P. Provero. Minimum physical length and the generalized uncertainty principle in string theory. Phys. Lett. B 234, 276 (1990).

9. E. Witten. Reflections on the fate of spacetime. Physics Today 49, 24 (1996).

10. M. Maggiore. A generalized uncertainty principle in quantum gravity. Phys. Lett. B 304, 65 (1993).

11. L.G. Garay. Quantum gravity and minimum length. Int. J. Mod. Phys. A 10, 145 (1995).

12. S. Capozziello, G. Lambiase, G. Scarpetta. Generalized uncertainty principle from quantum geometry. Int. J. Theor. Phys. 39, 15 (2000).

13. A. Kempf, G. Mangano, R.B. Mann. Hilbert space representation of the minimal length uncertainty relation. Phys. Rev. D 52, 1108 (1995).

14. F. Brau. Minimal length uncertainty relation and hydrogen atom. J. Phys. A 32, 7691 (1999). 
15. S. Das, E.C. Vagenas. Phenomenological implications of the generalized uncertainty principle. Can. J. Phys. 87, 233 (2009).

16. S. Hossenfelder. Minimal length scale scenarios for quantum gravity. Living Rev. Rel. 16, 2 (2013).

17. A. Tawfik, A. Diab. Generalized uncertainty principle: approaches and applications. Int. J. Mod. Phys. D 23, 1430025 (2014).

18. A. Tawfik, A. Diab. A review of the generalized uncertainty principle. Rep. Prog. Phys. 78, 126001 (2015).

19. R.C.S. Bernardo, J.P.H. Esguerra. Euclidean path integral formalism in deformed space with minimum measurable length. J. Math. Phys. 58, 042103 (2017).

20. C. Bambi, F.R. Urban. Natural extension of the generalised uncertainty principle. Class. Quant. Grav. 25, 095006 (2008).

21. R. Arnowitt, S. Deser, C.M. Misner. The dynamics of general relativity. In: Gravitation: An Introduction to Current Research, ed. by L. Witten, (Wiley, 1962) [ISBN: 9781114291669].

22. K.V. Kuchař, C.G. Torre. Gaussian reference fluid and interpretation of quantum geometrodynamics. Phys. Rev. D 43, 419 (1991).

23. J.A. Wheeler. Superspace and the nature of quantum geometrodynamics. In: Battelle Rencontres, eds. by C. DeWitt, J.A. Wheeler, (Benjamin, 1968).

24. B.S. DeWitt. The quantization of geometry. In: Gravitation: An Introduction to Current Research, ed. by L. Witten, (Wiley, 1962) [ISBN: 978-1114291669].

25. C.J. Isham. Structural issues in quantum gravity. arXiv:grqc/9510063.

26. J.D. Brown, D. Marolf. On relativistic material reference systems. Phys. Rev. D 53, 1835 (1996).

27. V.E. Kuzmichev, V.V. Kuzmichev. The Big Bang quantum cosmology: The matter-energy production epoch. Acta Phys. Pol. B 39, 979 (2008).

28. V.E. Kuzmichev, V.V. Kuzmichev. Quantum evolution of the very early universe. Ukr. J. Phys. 53, 837 (2008).

29. V.E. Kuzmichev, V.V. Kuzmichev. Quantum corrections to the dynamics of the expanding universe. Acta Phys. Pol. $B$ 44, 2051 (2013).

30. V.E. Kuzmichev, V.V. Kuzmichev. Can quantum geometrodynamics complement general relativity? Ukr. J. Phys. 61, 449 (2016).
31. V.E. Kuzmichev, V.V. Kuzmichev. The matter-energy intensity distribution in a quantum gravitational system. Quantum Stud.: Math. Found. 5(2), 245 (2018).

32. P.A.M. Dirac. The theory of gravitation in Hamiltonian form. Proc. Roy. Soc. A 246, 333 (1958).

33. F. Karolyhazy. Gravitation and quantum mechanics of macroscopic object. Nuovo Cimento A 42, 390 (1966).

34. M. Maziashvili. Space-time in light of Károlyházy uncertainty relation. Int. J. Mod. Phys. D 16, 1531 (2007).

35. W.G. Unruh. Why study quantum theory? Can. J. Phys. 64, 128 (1986).

36. G. Amelino-Camelia, L. Freidel, J. Kowalski-Glikman, L. Smolin. The principle of relative locality. Phys. Rev. D 84, 084010 (2011).

37. Lay Nam Chang, Z. Lewis, D. Minic, T. Takeuchi. On the minimal length uncertainty relation and the foundations of string theory. Advances in High Energy Physics 2011, 493514 (2011).

Received 21.11.18

В.Є. Кузъмичов, В.В. Кузъмичов

УЗАГАЛЬНЕНИЙ ПРИНЦИП

НЕВИЗНАЧЕНОСТІ У КВАНТОВІЙ КОСМОЛОГІЇ

ДЛЯ МАКСИМАЛЬНО СИМЕТРИЧНОГО ПРОСТОРУ

$\mathrm{P}$ е $з$ ю м е

У рамках канонічної квантової теорії, що бере до уваги гравітацію, для випадку максимально симетричного простору отримано нове співвідношення невизначеності. Це співвідношення встановлює зв'язок між флуктуаціями величин, що визначають внутрішню та зовнішню кривизни просторово-подібної гіперповерхні, та запроваджує принцип невизначеності для квантових гравітаційних систем. Запропоновано узагальнене співвідношення невизначеності для часу та енергії, що враховує гравітацію. Показано, що відоме співвідношення невизначеності Анру випливає 3 нового співвідношення невизначеності як окремий випадок. Як приклад, в рамках моделі, що має точний розв'язок, обчислені розміри флуктуацій масштабного фактора та спряженого імпульсу. Всі відомі видозміни принципу невизначеності, що раніше були виведені в різних підходах у теорії гравітації та теорії струн, були отримані як особливі випадки запропонованого загального виразу. 